\title{
Busca ativa de casos de leishmaniose cutânea em humanos e cães em área periférica do município de Campo Mourão - PR, Brasil
}

\author{
Active search of cutaneous leishmaniasis in humans and canines in the peripherical area of \\ Campo Mourão-PR-Brazil
}

\author{
Luís Antonio Sangioni' ${ }^{I}$ Cláudia Maria dos Santos GebaraII Geandra Meirice Aragão ${ }^{\text {III }}$ \\ Carlos Alberto de Andrade Bezerra ${ }^{\text {IV }}$ Crysthianne Cônsulo de Almeida ${ }^{I V}$
}

\section{-NOTA-}

\section{RESUMO}

Foi realizada uma busca ativa de casos de leishmaniose cutânea em humanos e cães, em área periférica da cidade de Campo Mourão - PR. No período de 01 a 30 de junho de 2004, uma equipe multidisciplinar visitou todas as residências e estabelecimentos comerciais situadas até $150 \mathrm{~m}$ da margem superior e inferior do rio 119, que delimita o município. Todos os casos humanos suspeitos de Leishmaniose Tegumentar Americana (LTA) foram encaminhados para o Posto de Saúde de referência para receberem os devidos tratamentos e orientação profilática. Foram encontrados 6 (seis) casos de pessoas suspeitas de LTA, sendo que 04 (quatro) resultaram em diagnóstico negativo e 02 (dois) em diagnóstico positivo. Nenhum cão apresentou lesão suspeita de LTA.

Palavras-chave: Leishmaniose cutânea, Leishmania, busca ativa, cão, Campo Mourão.

\section{ABSTRACT}

An active search was done to identify cases of leishmaniasis in humans and dogs in a peripherical area of Campo Mourão, PR, Brazil. From the first to thirtieth June of 2004, a multidisciplinary professional team visited all residences located within 150 meters from the superior and inferior margins of the river 119, which delimits the city. All human cases of American Tegumentary Leishmaniasis (ATL) were directed to reference health care center for the appropriated treatment and prophylactic orientation. Six cases of persons suspected of ATL were found; four patients resulted in a negative diagnosis while two cases were positive. No dogs demonstrated lesions that were suggestive of ATL.
Key words: cutaneous Leishmaniasis, Leishmania, active search, dogs, Campo Mourão.

A Leishmaniose Tegumentar Americana (LTA) é uma doença primariamente zoonótica, nãocontagiosa, causada por diversos protozoários do gênero Leishmania, envolvendo uma grande variedade de mamíferos silvestres, reservatórios do parasito, transmitida por insetos vetores da família Psychodidae, dos gêneros Lutzomya e Phlebotomus (MATTOS JR et al., 2004). As leishmanioses acometem mundialmente cerca de 1,5 milhões de pessoas por ano. Atualmente, 12 milhões apresentam alguma forma da doença e 350 milhões estão expostos a ela (DESJEUX, 2001). A LTA é originalmente silvestre, mas vem ocorrendo em áreas rurais e urbanas. A doença ocorre em locais onde há derrubada de matas e colonização recente, mas ultimamente vem sendo verificada também em áreas de colonização antiga, onde existem matas residuais ou de segunda formação (MARTINS et al., 2004). Segundo ZANZARINI et al. (2005), a LTA é amplamente distribuída no Brasil, ocorrendo em todos os Estados, com notificação em 276 dos 399 municípios pesquisados. O perfil da leishmaniose no Estado do Paraná não diferiu daquele assinalado para as outras

'Departamento de Medicina Veterinária Preventiva (DMVP), Centro de Ciências Rurais (CCR), Universidade Federal de Santa Maria (UFSM). Av. Roraima, 1000, Cidade Universitária, Camobi, Santa Maria, RS, Brasil. E-mail: sangioni@smail.ufsm.br. Autor para correspondência.

"Departamento de Medicina Veterinária, Centro Integrado de Ensino Superior, Campo Mourão, PR, Brasil

IIISecretaria de Abastecimento do Estado do PR (SEAB).

IV Prefeitura Municipal de Campo Mourão, PR, Brasil. 
regiões. A importância vetorial dos flebotomíneos exige intensivas investigações da ecologia destes dípteros como parte integrante da rotina dos serviços de saúde pública, sobretudo nas regiões onde as leishmanioses são endêmicas (TEODORO et al., 2003).

A presença de cães com infecção por $\boldsymbol{L}$. braziliensis tem sido constatada em áreas rurais onde a LTA se mantém endêmica e associada a ambientes alterados. Por causa da importância do cão como reservatório da leishmaniose, a Fundação Nacional de Saúde (FUNASA) adota no Brasil, como medida de controle em áreas endêmicas, a eliminação desses animais quando são soropositivos para Leishmania chagasi (SILVA et al., 2005).

A doença no homem abrange desde formas inaparentes, lesões discretas de pele que podem evoluir espontaneamente para cura, ulcerações múltiplas, lesões de mucosas, até formas com tendência às metástases e recidivas, de curso lento e tratamento difícil, com a presença de seqüelas desfigurantes, destrutivas e incapacitantes (RAZIERA et al., 2005). O diagnóstico laboratorial em seres humanos da LTA pode ser realizado pela pesquisa direta do parasito em material obtido de úlceras através da intradermorreação de Montenegro e da pesquisa de anticorpos antiLeishmania no soro sangüíneo (SILVEIRA et al., 1996).

Uma das formas de descoberta de novos casos de doenças é a busca ativa, que consiste na visita de técnicos às residências e no encaminhamento dos casos suspeitos aos profissionais de saúde. Os métodos ativos são mais fidedignos, embora sejam mais trabalhosos e demandem maior tempo. Outra vantagem da metodologia é a necessidade de um número maior de visitas aos locais onde se encontram os pacientes, proporcionando maior oportunidade de interações sociais. As técnicas ativas não apenas permitem uma detecção de casos, mas também permitem visitar as áreas suspeitas regularmente e monitorar os possíveis problemas que possam ocorrer na localidade (MINISTÉRIO DA SAÚDE, 2000).

O objetivo deste trabalho foi observar a ocorrência da LTA em populações humana e canina domiciliadas às margens do rio 119, no período de 01 a 30 de junho de 2004, no município de Campo Mourão, localizado a $24^{\circ} 02^{\prime} 38^{\prime \prime}$ S e $52^{\circ} 22^{\prime} 40^{\prime \prime} \mathrm{O}$, com clima subtropical úmido mesotérmico. As médias anuais de temperatura giram em torno de $23^{\circ} \mathrm{C}$, com invernos brandos entremeados de períodos de seca. A equipe multidisciplinar foi composta por 28 agentes de combates a endemias, 65 agentes comunitários, 01 Enfermeira, 03 Médicos Veterinários, 05 acadêmicos do curso de Medicina Veterinária do Centro Integrado de Ensino Superior (CIES) e 01 Médico Dermatologista. A área selecionada para as visitas apresentava-se degradada e com as matas ciliares depredadas. As áreas limítrofes foram ocupadas com plantações de soja, trigo, milho, consorciadas com áreas de pastagens.

Todas as residências e estabelecimentos comerciais situadas até $150 \mathrm{~m}$ da margem superior e inferior do rio 119 foram visitadas. Nos pacientes humanos suspeitos, foi realizada a Reação Intradérmica de Montenegro, sendo encaminhados em seguida para o Posto de Saúde de referência para confirmação de diagnóstico e para receberem os devidos tratamentos e a orientação profilática. Foram encontrados seis casos de pessoas suspeitas de LTA, sendo que quatro resultaram em diagnóstico negativo e dois em diagnóstico positivo. Os testes intradérmicos apresentaram reações maiores que $05 \mathrm{~mm}$ de diâmetro após 48h de inoculação do antígeno. As principais lesões dérmicas encontradas nos humanos estavam localizadas na face, compatíveis com lesões provocadas por LTA. Os cães domiciliados foram avaliados clinicamente para a presença de lesões características, não sendo observados animais com lesões suspeitas. Foram visitadas 2.161 residências e 67 estabelecimentos comerciais, totalizando 2.218 unidades, em 153 quadras urbanas e 49 rurais. A média de pessoas habitadas por imóvel era de 4,35. As notificações de LTA, nos últimos cinco anos, no município de Campo Mourão, tem variado de um a quatro com a média de 2,4 casos. Segundo SILVEIRA et al. (1996), os casos de LTA no norte do Estado vêm aumentando nos últimos anos. No Paraná, existem diferentes focos de LTA com diferentes biótipos, sendo a Lutzomya intermedia e a L. whitmani incriminadas como os principais vetores (CASTRO et al., 2005).

Os reservatórios primários do parasito são os animais silvestres, porém os animais que convivem diretamente com o homem, como o cão e o cavalo, servem secundariamente como reservatórios. A presença de animais, mesmo sadios, próximos ao domicílio representa potenciais reservatórios ou fonte de alimentação para o inseto vetor (MOREIRA et al., 2002). AMPUERO et al. (2005) relataram que os indivíduos infectados em uma residência podem atuar como reservatórios intradomiciliares do protozoário, servindo como fonte de infecção para os flebotomíneos adaptados a esse meio ambiente. A presença de cães no domicílio tem sido relatada como fator de risco para LTA, podendo esses animais comportarem-se como hospedeiros acidentais, assim como os seres humanos, conforme descrito nesse estudo. O desequilíbrio ambiental desencadeado pelo desmatamento e pela expansão de áreas agricultáveis em meio silvestre podem conduzir a alterações da densidade de vetores 
e a possíveis reservatórios da doença (DUJARDIN, 2006), o que também foi observado nesse estudo.

A prevenção da LTA no extradomicílio na área periurbana de Campo Mourão, como em outras regiões do Brasil, tem-se constituído num problema difícil de resolução, seja pela amplitude dos espaços a serem atuados e pela insuficiência de conhecimentos sobre as reais condições de transmissão.

\section{REFERÊNCIAS}

AMPUERO J. et al. Factores de riesgo para la transmisión de leishmaniasis cutánea en niños de 0 a 5 años en un área endémica de Leishmania (Viannia) braziliensis. Cadernos de Saúde Pública, v.21, n.1, p.353-355, 2005.

CASTRO E.A. et al. Eco-epidemiological survey of Leishmania (Viannia) braziliensis american cutaneous and mucocutaneous leishmaniasis in Ribeira Valley River, Parana state, Brazil. Acta Tropica, n.93, p.141-149, 2005

DUJARDIN, J.C. Risk factors in the spread of leishmaniases: towards integrated monitoring? Trends In Parasitology, v.22 n.1, p.1771-1773, 2006.

DESJEUX, P. The increase in risk factors for leishmaniasis worldwide. Transactions of the Royal Society of Tropical Medicine and Hygiene, n.95, p.239-243, 2001

MARTINS, M.L. et al. Ecoepidemiologia da leishmaniose tegumentar no município de Buriticupu, Amazônia do Maranhão, Brasil, 1996 a 1998. Cadernos de Saúde Pública, v.20, n.3, p.735-743, 2004.

MATTOS JR D.G. et al. Aspectos clínicos e de laboratório de cães soropositivos para leishmaniose. Arquivo Brasileiro de
Medicina Veterinária e Zootecnia, v.56, n.1, p.119-122, 2004.

MINISTÉRIO DA SAÚDE, Fundação Nacional de Saúde. Gerência técnica de doenças transmitidas por vetores e antropozoonoses. São Paulo, 2000. 61p. (Manual de controle da Leishmaniose Tegumentar Americana).

MOREIRA, R.C. et al. Awareness of american tegumentary leishmaniasis (ATL) and use of alternative therapies in an endemic area in the Amazon region in the state of Maranhão, Brazil. Cadernos de Saúde Pública, v.18, n.1, p.187-195, 2002

RAZEIRA, F. et al. Leishmaniose tegumentar americana: primeiros casos autóctones notificados no Rio Grande do Sul desde 2001 e revisão da literatura. Revista AMRIGS, v.4, n.3, p. 165-170, 2005.

SILVA, A.V.M. et al. Leishmaniose em cães domésticos: aspectos epidemiológicos. Cadernos de Saúde Pública, v.21, n.1, p.324-328, 2005.

SILVEIRA, T.G.V. et al. Aspectos epidemiológicos da leishmaniose tegumentar em área endêmica do estado do Paraná, Brasil. Cadernos de Saúde Pública, v.12, n.2, p.141-147, 1996.

TEODORO, U. et al. Influência da reorganização, da limpeza do peridomicílio e a da desinsetização de edificações na densidade populacional de flebotomíneos no Município de Doutor Camargo, Estado do Paraná, Brasil. Cadernos de Saúde Pública, v.19, n.6, p.1801-1813, 2003.

ZANZARINI, P.D. et al. Leishmaniose tegumentar americana canina em municípios do norte do Estado do Paraná, Brasil. Cadernos de Saúde Pública, v.21, n.6, p.957-1961, 2005. 